\title{
Comparison Between Aqueous and Nonaqueous AOT-Heptane Reverse Micelles Using Acridine Orange as Molecular Probe
}

\author{
R.D. Falcone, N.M. Correa, M.A. Biasutti and J.J. Silber \\ Departamento de Quimica, Universidad Nacional de Río Cuarto. Agencia N ${ }^{0} 35800$ Rio Cuarto, Ar- \\ gentina \\ E-mail: jsilber@exa.unrc.edu.ar
}

\begin{abstract}
Aqueous and nonaqueous AOT/n-heptane reverse micelles where characterized by UV-Visible and fluorescence spectroscopy of AO. The study shows the presence of the dimer in aqueous reverse micelles which is not present in the nonaqueous systems. It seems that there is a conversion of the dimer to monomer in the aqueous reverse micelles at high AOT concentration. In the nonaqueous systems, there is only partition of the monomer. The apparent constants of these processes were calculated.
\end{abstract}

\section{Introduction}

Reverse micelles are aggregates of surfactant molecules with their polar groups concentrated in the interior while the hydrophobic moieties extend into and are surrounded by, the bulk apolar solvent. Thus, water or other polar solvent with high dielectric constants and unmiscible in the hydrocarbon solvent such as ethyleneglycol (EG), N,N-Dimethylformamide (DMF), glycerol (GY), Propileneglycol (PE) can be solubilized in the polar core. The last ones are called nonaqueous microemulsions and present a series of advantages over the aqueous reverse micelles for example can be useful media for organic reaction such as Diels- Alder [1] and others.

The microemulsions can be characterized by using molecular probes such as: 1-methyl-8-oxyquinolinium betaine $(\mathrm{QB})$ or the free base of the dye acridine orange AO [2]. The aim of the present contribution is to investigate the properties of the base AO in aqueous and nonaqueous AOT/n-heptane microemulsions. Thus, the spectroscopic behavior of AO in AOT/n-heptane using water, DMF and EG as polar solvents has been studied by the absorption and fluorescence spectra.

\section{Experimental}

Sodium 1,4-bis(2-ethylhexyl) sulfosuccinate (AOT) from SIGMA was dried under vacuum over $\mathrm{P}_{2} \mathrm{O}_{5}$. The molar ratio between polar solvent and AOT is defined as $\mathrm{W}_{\mathrm{s}}=$ [polar solvent $] /[\mathrm{AOT}]$. The free base of AO from SIGMA was used as received. The polar solvent ethyleneglycol (EG) and N,N- 
Dimethylformamide (DMF) all from ALDRICH (more than 99\% of purity) were used without further purification. Ultrapure water was obtained from Labonco equipment model 90901-01.

\section{Results and discussion}

The absorption spectra of $\mathrm{AO}$ in water [3] at $\mathrm{pH}<10$ (cationic form, $\mathrm{AOH}^{+}$), show two bands, at 468 and other at $490 \mathrm{~nm}$ which can be attributed to the dimer and monomer specie respectively. At $\mathrm{pH}$ $>10$ (basic form) only the monomer's band is present. Thus, only $\mathrm{AOH}^{+}$is the species that can suffer the dimerization process.

In aqueous reverse micelles systems, there are four processes that $\mathrm{AO}$ can suffer, a) distribution between the organic phase and the micellar interface; b) protonation to give $\mathrm{AOH}^{+}$; c) dimerization of $\mathrm{AOH}^{+}$and d) conversion of the dimer to monomer by the micelle. The spectra of AO with AOT concentration show the disappearance of the band originally present in n-heptane $(\lambda=417 \mathrm{~nm})$ and the appearance of the dimer band at $462 \mathrm{~nm}$. The monomer band appears at [AOT] $>3.4 \times 10^{-4} \mathrm{M}$. There are two isosbestic points, at 427 and $467 \mathrm{~nm}$. The fluorescence spectra show the band of the dimer at 644 $\mathrm{nm}$ at low [AOT] and the band of the monomer at $544 \mathrm{~nm}$. On the other hand, in the nonaqueous microemulsion, $\mathrm{AOH}^{+}$was not detected and only the distribution of the $\mathrm{AO}$ between the two pseudophases is observed. The absorption and the emission spectra are consistent with these facts.

The value of the distribution constant, $K_{\text {dist }}$ for the process a) was calculated by the Ketelaar approach [4] and, the value of the dimer dissociation $K_{\text {des }}$ for the process d) was calculated by the method showed in Ref [5].

The values of $K_{\text {dist }}$ show the following order: $K_{\text {dist }}{ }^{\text {Water }}>K_{\text {dist }}{ }^{E G}>K_{\text {dist }}$ DMF. This can be explained considering the micropolarity of the interfaces and hydrogen bond interactions.

Acknowledgments: We gratefully acknowledge the financial support from CONICET, CONICOR, FONCYT, SECyT UNRC.

\section{References and Notes}

1. Ray, S.; Moulik, S.P. Langmuir 1994, 10, 2511.

2. Falcone, R.D.; Correa, N.M.; Biasutti, A.; Silber, J.J. Atualidades Fisquim. Org. 1998, 196.

3. Zanker, V. Z. Phys. Chem. 1952, 199, 225.

4. Ketelaar, J.A.A.; Van de Stolpe, C.; Gersmann, H.R. Rec. Trav. Chim. 1951, 70, 499.

5. Correa, N M.; Silber, J.J. J. Molec. Liquids 1997, 72, 163. 